\title{
Cohesión social transnacional y migración en Guanajuato. Construyendo una perspectiva desde el Sur
}

\section{Transnational social cohesion and migration in Guanajuato. A perspective in construction from the South}

Recibido el 23 de marzo de 2020. Aceptado el 15 de octubre de 2020. Publicado el 21 de octubre de 2020.

*Autora para correspondencia: Ana Vila Freyer. Correo electrónico: ana6509@yahoo.com

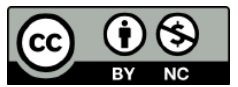

Esta obra está protegida bajo una Licencia Creative Commons Atribución-NoComercial 4.0 Internacional.
Ana Vila Freyer ${ }^{\text {** }}$ (D) https://orcid.org/0000-0002-3199-0604

${ }^{a}$ Universidad Latina de México, Celaya, Guanajuato, México, correo electrónico: ana6509@yahoo.com

\section{Resumen}

¿Cómo afectan los grupos migrantes la cohesión social, los valores comunitarios y el desarrollo de sus comunidades de origen? Para responder estas preguntas se aplicó una encuesta exploratoria a 658 personas, $21 \%$ de las cuales eran personas migrantes retornadas y/o sus familias. El objetivo fue identificar los recursos movilizados por los migrantes durante su trayectoria migratoria con base en las cinco dimensiones que según Jane Jenson componen la cohesión social. Este trabajo tuvo dos hallazgos: los grupos migrantes han sostenido sus valores y compromisos comunitarios sobre una estructura de vínculos sociales apoyada en relaciones familiares y de amistad, así como en un bajo reconocimiento a las instituciones políticas nacionales y subnacionales. La evidencia sugiere, además, que algunos elementos de participación comunitaria que constituyen esta estructura de vínculos se manifiestan de manera simultánea en espacios geográficos múltiples. Esto reforzaría un sentido de pertenencias múltiples y crearía una noción de cohesión social transnacional y una agenda de investigación que lo verifique.

Palabras clave: cohesión social, comunidad, pertenencias múltiples, transnacionalismo, migración internacional, retorno.

\section{Abstract}

How do migrant groups affect social cohesion, community values and the development of their communities of origin? To answer these questions, an exploratory survey was applied to 658 people, $21 \%$ of whom were returned migrants and/or their families. The objective was to identify the resources mobilized by migrants during their migratory trajectory based on the five dimensions that, according to Jane Jenson compose social cohesion. This work had two findings: migrant groups

CÓMO CITAR: Vila Freyer, A. (2020). Cohesión social transnacional y migración en Guanajuato. Construyendo una perspectiva desde el Sur [Transnational social cohesion and migration in Guanajuato. A perspective in construction from the South]. Estudios Fronterizos, 21, e059. https://doi.org/10.21670/ref.2017059 
have sustained their community values and commitments in a structure of social linkages supported by family and friendship relationships, as well as a low recognition of national and subnational political institutions. Evidence also suggests that some elements of community participation that constitute this linkage structure are present simultaneously in multiple geographic spaces. This would reinforce a sense of multiple belonging and create a notion of transnational social cohesion and a research agenda to verify it.

Keywords: social cohesion, community, multiple belonging, transnationalism, international migration, return.

\section{Introducción}

Este trabajo busca explicar la forma cómo los migrantes han constituido vínculos comunitarios transnacionales desde un punto de vista de cohesión social. El tema contiene una paradoja: ya que mientras que el transnacionalismo es concebido como los contactos simultáneos y permanentes que mantienen a los migrantes conectados con las comunidades de origen y destino (Glick Schiller et al., 1992), la cohesión social hace énfasis, desde la perspectiva de los países de acogida, en los efectos económicos, sociales o culturales que resaltan la dificultad de asimilar a los migrantes dentro del orden social establecido. La primera perspectiva busca explicar la forma como los migrantes viven su vida a través de las fronteras que conectan dos países simultáneamente; la segunda hace énfasis en la integración de comunidades nacionales, así como en los elementos que sostienen el tejido social nacional y que, por tanto, constituyen una sociedad bien ordenada (Glick Schiller et al., 1992; Jenson, 1998; Ratcliffe, 2012; Portes \& Vickstrom, 2012). Al utilizar una perspectiva histórica, seguiremos las estructuras de vinculación que tienen los migrantes con sus comunidades de origen, para discutir que en las comunidades rurales del sur de Guanajuato existen grupos sociales que han constituido una forma de cohesión social transnacional.

Por cohesión social transnacional nos referimos a grupos sociales móviles que han tejido redes densas (Coleman, 1998) en las que soportan su sentido de pertenencia comunitarios y han establecido valores compartidos en espacios geográficos múltiples. Ello nos permite proponer las hipótesis de que sus formas de asociación y la representación subjetiva con la que reconstituyen su pertenencia comunitaria (Dickes et al., 2011; López Sánchez, 2016) se han basado en dos puntos: 1) una estructura de vínculos sociales sostenida fundamentalmente en vínculos familiares y de amistad, así como en la baja confianza en los recursos político-institucionales (Portes \& Vickstrom, 2012) existentes en Guanajuato, primero, y en México, después; 2) la estructura de vínculos sociales se ha manifestado históricamente de manera simultánea en las interacciones individuales y familiares existentes en espacios geográficos múltiples, lo que refuerza un sentido de pertenencia multiespacial.

El concepto de cohesión social que explora este trabajo es el definido por Jenson (1998, p. 13): "la cohesión social es un proceso que sostiene el orden social en el que los individuos mantienen valores compartidos y un compromiso hacia su comunidad". Esta definición tiene como unidad de análisis la comunidad nacional y su preocupación central está dada por el proceso en el que se sostiene la integración del tejido social dentro de un espacio geográficamente delimitado por las fronteras. A pesar de tomar como base esta definición, al enfocar el análisis en poblaciones móviles es necesario 
cuestionar ¿cómo el movimiento espacial de un grupo social afecta ese proceso de integración comunitaria? ¿cómo este grupo móvil mantiene los valores comunitarios y establece compromisos en sus comunidades antes de migrar, durante el proceso migratorio y al regresar a sus comunidades de origen? ¿cómo afectan su sentido de pertenencia comunitaria? Es decir, en lugar de ser una característica propia de espacios nacionales cerrados, en cuanto están delimitados por las fronteras, se analizan las estructuras de vínculos de los grupos migrantes en espacios transnacionales. Las estructuras de vinculación son consideradas como los atributos, relaciones y recursos individuales que conforman los grupos sociales y estos, a su vez, determinan las relaciones entre individuos y grupos sociales, así como de individuos y sociedad (Dickes et al., 2011). Esto nos permite asumir que estas estructuras de vinculación se mantienen a pesar de la división dada por fronteras, al establecer un proceso que explica cómo las personas y grupos migrantes configuran sus relaciones con el mercado, con sus familias y con sus comunidades en contextos multiespaciales. También en la forma como las personas migrantes establecen ciudadanías incompletas al estar ausentes de los países de origen, y tener derechos muy limitados en los países de destino. Ya que, como señala Anderson (2019, p. 2), "es posible distinguir entre quién es el migrante en la ley y en las políticas migratorias, el migrante en las estadísticas migratorias y el migrante en el debate público".

$\mathrm{El}$ argumento se construye con base en un trabajo de campo realizado en el verano de 2016 que examinaba las siguientes preguntas: ¿cómo los migrantes construyen su sentido de pertenencia comunitario al mantener una vida multiespacial? ¿cómo establecen sus actividades de socialización política, económica y cultural en ambos países? y, finalmente, ¿cómo han adaptado y readaptado su vinculación comunitaria en el espacio y en el tiempo? Al hacer énfasis en la forma como estos elementos se han construido históricamente en la percepción de los migrantes, en sus interacciones cotidianas antes de migrar, durante su estancia en otro país y al regresar a sus comunidades de origen (Eckstein, 2015) se identificó un orden social basado en una estructura de vinculaciones que se mantiene a través del tiempo de manera multiespacial. Esto implica que, el movimiento migratorio se sostiene en un proceso específico de relaciones sociales en el que los valores compartidos, compromisos comunitarios y sentidos de pertenencia se alternan cotidianamente en tiempos y espacios distintos. Para presentar nuestro argumento en el siguiente apartado se discuten los conceptos básicos utilizados en el cuestionario, así como la metodología utilizada. En el tercer apartado presentaremos los resultados del trabajo de campo, lo que nos llevarán a la discusión y conclusiones.

\section{Cohesión social en el régimen migratorio México-Estados Unidos: notas metodológicas}

Una de las presunciones de este trabajo es que la centenaria migración México-Estados Unidos ha estructurado diferentes arreglos o regímenes institucionales que, de manera formal o informal, han guiado las condiciones políticas, económicas y sociales, así como las políticas migratorias nacionales que corresponden a cada uno de los seis periodos identificados por Durand (2016) que han caracterizado este proceso a lo 
largo de sus más de 100 años de historia. ${ }^{1}$ De alguna manera han establecido un orden social en el que los individuos mantienen valores compartidos y un compromiso hacia su comunidad (Jenson, 1998, p. 13) que alternan en distintos espacios geográficos y comunidades (Glick Schiller et al., 1992). En este proceso han establecido una estructura de vínculos económicos, políticos y sociales que pueden identificarse en las cinco dimensiones analíticas que conforman la cohesión social (Jenson 1998, p. 15-17; Jenson, 2010): pertenencia/aislamiento; inclusión/exclusión; participación/apatía; reconocimiento/rechazo; legitimidad/ilegitimidad.

La dimensión de la pertenencia/aislamiento se refiere a los sentimientos compartidos por las comunidades que facilitan la integración de los individuos. Una amenaza a la cohesión social "está asociada con los sentimientos de aislamiento de la comunidad" (Jenson, 1998, p. 15-17; Jenson, 2010). La dimensión de inclusión/exclusión está relacionada con los mercados. Una sociedad bien cohesionada asegura la inclusión, según la autora, e implica la igualdad de oportunidades, especialmente en los mercados laborales, para crear condiciones de cohesión; por tanto, un riesgo a la cohesión social está entonces asociada con la exclusión del mercado. La dimensión participación/apatía tiene que ver con las diferentes formas de interacción y organización comunitaria para hacer valer sus intereses ante los gobiernos nacionales y subnacionales (Jenson, 1998, p. 15-17; Jenson 2010). La cuarta dimensión, reconocimiento/rechazo, es con la que se establece (Jenson, 1998, p. 15-17) "la necesaria mediación de las diferencias sobre el poder, los recursos y los valores que es asegurada por instituciones, sean formales o informales, públicas o privadas (...) que en distintos lugares o momentos pueden ser mediadores o promotores de conflictos". De tal manera que el mantenimiento de la cohesión social depende de la creación de instituciones que contribuyan, en lugar de socavar, al reconocimiento de la diferencia. El reconocimiento "definido como la sensación de los ciudadanos de que los otros los aceptan y reconocen sus contribuciones a la comunidad" (Jenson, 1998, p. 15-17); mientras que el rechazo y la intolerancia, así como los esfuerzos por lograr un excesivo nivel de unanimidad, hacen a los Estados y comunidades menos "vivibles". La última dimensión, legitimidad/ilegitimidad, Jenson la define como el papel fundamental de la mediación política (Jenson, 1998, p. 15-17; 2010). En concreto se refiere a la capacidad del Estado de gestionar el conflicto en una sociedad plural a través de "macroinstituciones" que garantizan seguridad, acceso a programas sociales y servicios necesarios a comunidades diversas. Este grupo de organizaciones intermediarias incluyen servicios sociales, partidos políticos, agencias gubernamentales, así como organismos de la sociedad civil, entre otros.

Esta perspectiva de la cohesión social contiene elementos que definen quién forma parte de la comunidad, así como valores compartidos que se construirían en espacios sociales cerrados y delimitados por fronteras. Utilizamos estas cinco dimensiones para construir los reactivos de un cuestionario cerrado y los temas de entrevistas semiestructuradas. Estos elementos nos ayudaron a identificar de manera comparativa la forma como los migrantes perciben sus vínculos comunitarios antes de salir de

\footnotetext{
${ }^{1}$ La idea de régimen migratorio tratamos de derivarla de la noción de régimen de ciudadanía definido por Jenson y Saint Martin (2003, p. 80): "By the concept of citizenship regime we mean the institutional arrangements, rules and understandings that guide and shape concurrent policy decisions and expenditures of states, problem definitions by states and citizens, and claims-making by citizens". ("Por el concepto de régimen de ciudadanía nos referimos a los arreglos institucionales, reglas y entendimientos que guían y dan forma a las decisiones políticas concurrentes y a los gastos de los estados, a las definiciones de los problemas por los estados y los ciudadanos, y a las reivindicaciones de los ciudadanos" [traducción libre]).
} 
México, durante su estancia en Estados Unidos (EUA) y al regresar a México. Entre ellos, nos enfocamos en sus condiciones de inclusión al mercado, su percepción del sentido de pertenencia y los problemas vividos para formalizarla al regresar o de vivir de manera irregular en EUA, su participación en organizaciones sociales y políticas, el reconocimiento y pertenencia de sus comunidades y la legitimidad/confianza manifestada hacia las instituciones públicas. Hacer una comparación en el tiempo, en los casos en que era posible, permitió identificar la manera como estas estructuras de vinculación se mantienen y amplían en forma multiespacial, combinando la experiencia local, nacional y transnacional.

La encuesta se aplicó a la persona migrante o a un familiar cercano a esta como su esposa, alguno de sus padres o sus hijos. El objetivo era identificar la forma en que la persona migrante se sentía acogida por otros miembros de la comunidad, cómo y cuánto tiempo después de regresar a México se incluyó al mercado y en qué condiciones, cómo participaba en las actividades comunitarias. Finalmente, buscamos detectar la forma en que la confianza o desconfianza en sus vecinos, en distintas agencias gubernamentales o de salud les hacía acudir a ellas para solicitar ayuda, recuperar papeles de identidad de ellos o de sus hijos — sobre todo nacidos o regularizados en EUA- para acceder a servicios sociales y utilizar instituciones financieras. ${ }^{2}$ Los datos presentados aquí se reforzaron con siete entrevistas semiestructuradas en las que se incluyó la historia migratoria, así como los procesos y problemas de reintegración de los entrevistados jóvenes estudiantes de preparatoria y universidad de entre 15 a 23 años que detectamos durante el trabajo de campo-. Aunque en el documento utilicemos en ocasiones el concepto de migrante retornado, no es el interés de este proyecto enfocarnos en el migrante como alguien que ha concluido un ciclo migratorio, sino como un migrante sin papeles en Eua que en el momento de la entrevista había regresado a México. ${ }^{3}$

Los municipios de estudio fueron seleccionados comparando los resultados para Guanajuato del Índice de Intensidad Migratoria 2000 y 2010 (Consejo Nacional de Población [Conapo], 2001; 2012) en dos puntos: los hogares que declararon recibir remesas y los que manifestaron tener migrantes de retorno, en ambos casos la pregunta del censo se construye sobre los hogares que han recibido remesas o tienen migrantes de retorno durante los cinco años anteriores. De este modo, identificamos Apaseo el Alto como un municipio con alto retorno y alta recepción de remesas, en el que asumimos que los migrantes que regresaron fueron los familiares de un migrante económico-laboral y que este siguió en EuA enviando dinero a su familia, lo que explicaría la aparente contradicción del aumento de hogares que reportan retorno, con el incremento de hogares que reportan recibir remesas. Por su parte Tarimoro y Celaya, municipios en donde hay un alto retorno y bajó la recepción de remesas, asumimos que se dio o bien el retorno de la familia completa (Moctezuma, 2013; Vila Freyer, 2015) o el retorno del migrante económico-laboral, lo que explicaría la reducción de las remesas. En los tres municipios elegimos tres comunidades rurales con población que rondara entre 4000 y 5000 habitantes, con la excepción de Apaseo el Alto, en donde además aplicamos encuestas en la cabecera municipal que es una comunidad urbana con 98000 habitantes.

\footnotetext{
${ }^{2}$ Detectamos en el proceso a migrantes circulares que, al contar con papeles, pueden moverse fácilmente entre México y Estados Unidos y que por alguna razón estaban en México en el momento de la encuesta. Descartamos las respuestas recabadas a estas personas.

${ }^{3} \mathrm{~A}$ la pregunta ¿en qué año regresó la última vez?, 30.9\% regresó entre 1981 y 2006 y $69.1 \%$ entre 2007 y 2016, los años de la crisis económica global.
} 
Se trató de una encuesta exploratoria, ${ }^{4}$ en la que participaron 658 personas, que representaría aproximadamente $2 \%$ de la población de las comunidades rurales. Al ser comunidades rurales cuya población es atraída laboralmente hacia centros urbanos como Celaya y Querétaro, se dio prioridad a los días de mercado que es cuando se concentra la población en las plazas públicas. El levantamiento se realizó, por razones de seguridad, entre las 10 y las 15 horas, a personas que tenían entre 17 y 60 años. Del total de encuestados solamente $41.6 \%$ contaba con algún familiar retornado de EUA y, de este grupo, $42.6 \%$ de los encuestados fueron los propios migrantes que habían regresado, $98 \%$ de los cuales regresaron solos y $2 \%$ respondió haber regresado ya sea él/ella con toda su familia. Del $57.4 \%$ restante los que respondieron la encuesta fueron sus familiares directos, es decir, la esposa (23\%), los padres (15\%), alguno de sus hijos (7\%), u otro (10.4\%). Es importante destacar que $26.5 \%$ de los migrantes retornados habían regresado en los últimos tres años (a partir de 2014). De los migrantes retornados, $69 \%$ vivió entre uno y seis años fuera del país $(44 \%$ de uno a tres años), $16 \%$ menos de un año y $15 \%$ había vivido más de siete años en EuA.

De los encuestados, $59 \%$ eran mujeres y $41 \%$ hombres; $7 \%$ de los cuales eran analfabetos. De los respondientes, $24 \%$ tenían primaria terminada, $34 \%$ secundaria terminada, $19 \%$ preparatoria terminada y $5 \%$ universidad o universidad técnica terminada, el resto tenía los estudios truncos. Otro dato importante es que $20 \%$ se llevó a su familia con él, sea desde el inicio de la jornada migratoria (10.2\%), o cuando ya estaba instalado allá (9.96\%). Los demás nunca se llevaron a su familia. Regresó solo $84 \%$, regresó junto con su familia $13 \%$ y solamente $3 \%$ envió a su familia a México mientras el jefe del hogar continuó en EuA.

\section{Cohesión social transnacional en Guanajuato: una primera aproximación}

Guanajuato es un estado que se formó por migrantes. Desde la época colonial ha mantenido una fluctuación poblacional que ha tenido relación directa con las condiciones del mercado laboral interno (Guevara Sanginés, 2017). Desde inicios del siglo $\mathrm{xx}$, ha sido parte de la región tradicional expulsora de población por más de 100 años (Durand, 2016; Durand \& Massey, 2003). Al ser un estado con alta migración, 31 de sus 46 municipios registran una alta o muy alta relación con la migración (Conapo, 2012). Por ello, más de la mitad de los encuestados (56\%) respondió que ellos, o algún miembro de su familia, ha emigrado a EuA. De estos casos, lo primero que se detecta son los cambios generacionales vividos al especificar quién es el miembro de la familia que ha participado de la migración: $100 \%$ de los encuestados tienen o tuvieron un abuelo que emigró, de sexo masculino; cuando la persona migrante es el cónyuge, $86 \%$ es del sexo masculino y $14 \%$ del femenino; el padre era $79 \%$ de los casos. El porcentaje sigue cambiando al preguntar si la persona con experiencia migratoria es un hijo (77\%), una hija (23\%) o una madre (21\%). La migración pasó de ser un asunto estrictamente masculino, a uno en el que entre una cuarta y una quinta parte de las personas que participan en este proceso son mujeres.

\footnotetext{
${ }^{4}$ Uno de los objetivos de la encuesta era precisamente validar el cuestionario para aplicarlo con una muestra representativa en todo el estado.

${ }^{5}$ En Apaseo el Alto levantamos encuestas a $0.002 \%$ de la población, aproximadamente 158 encuestas.
} 
Detectamos también que, en la región sur de Guanajuato, el migrante está cada vez menos relacionado con el trabajo agrícola. Al cuestionar sobre el proyecto migratorio - la razón por la que se migró y el uso que darían a los salarios ganados en EUAmenos de $10 \%$ de las respuestas están relacionadas con el trabajo del campo, adquirir tierras o materiales agrícolas. Sin embargo, antes de migrar $11.3 \%$ tenía tierras, al regresar $27.4 \%$ las tenía; la adquisición de maquinaria para el campo pasó de $6.6 \%$ a $11.2 \%$ y la adquisición de ganado pasó de $3.7 \%$ a $13 \%$.

A continuación, presentamos los elementos específicos considerados en el trabajo de campo para cada una de las dimensiones de la cohesión social.

\section{Pertenencia/aislamiento: de la vida ilegal en Estados Unidos, a la vida ilegal en México}

Uno de los cambios más importantes vividos por los migrantes detectados en la encuesta es que al regresar se sienten más seguros al vivir en México que en EuA. Al preguntar si se sentían seguros viviendo en EUA, $58 \%$ dijo que sí; al hacer la misma pregunta sobre su vida actual las respuestas positivas suben a $67 \%$. Esta respuesta parece reflejar la certidumbre de vivir "regularmente" en su país, en un ambiente familiar y sin estar expuestos a redadas, ya que se trata fundamentalmente de migrantes en una situación irregular. ${ }^{6}$ Sin embargo, $26 \%$ de los entrevistados tuvieron problemas para regularizar sus papeles o los de sus hijos; mientras que $29 \%$ no había logrado tener acceso a servicios de salud. Como señaló una de las jóvenes entrevistadas:

Ser migrante no es malo en sí, a través de eso se aprenden experiencias buenas y malas (...) ser ilegal es como no tener una prueba de que resides ahí (...) es vivir con la preocupación de no hacerse notar, de no pasar cerca de policías o retenes. (...) $\mathrm{Al}$ regresar seguimos siendo ilegales un tiempo (...) mi mamá ya tenía mi acta y lo único que fue a renovarla (...) el problema que todavía está es el de mi hermana que tiene el acta estadounidense y había que hacer la mexicana (Ivonne, entrevista en Salvatierra, Guanajuato, 21 de abril de 2016).

La sensación de seguridad llama la atención si se toma en cuenta el incremento de la violencia en las comunidades del sur de Guanajuato que tiene relación con el crimen organizado y que empezó a incrementarse a partir de $2015 .^{7}$ Como se ve en la Tabla 1, es uno de los problemas que los migrantes padecen al regresar a México. Su sentido de pertenencia a la comunidad se ve afectado por la corrupción de las autoridades, la falta de servicios de salud, los problemas para arreglar papeles o porque

\footnotetext{
${ }^{6} \mathrm{~A}$ la pregunta precisa sobre el estatus migratorio presentado por las personas migrantes mientras estuvieron fuera la respuesta es que la mayoría se fue sin papeles: $79 \%$ el cónyuge, $66 \%$ el hijo, $53 \%$ el padre o madre. Sin embargo, los que tienen residencia o Green card - para poder circular por ambos paísesson $7 \%$ para el cónyuge, $16 \%$ para el hijo, $25 \%$ para el padre o madre y $50 \%$ para el abuelo. Las personas que han viajado cinco veces o más a EUA para trabajar: el cónyuge $27 \%$, el hijo $10 \%$, el padre/madre $22 \%$, el abuelo $25 \%$. Mientras que los que se fueron y ya no volvieron resultaron, en el mismo orden: $15 \%$ para el cónyuge, $45 \%$ para el hijo, $16 \%$ para el padre/madre y $50 \%$ para el abuelo. De los entrevistados, $9 \%$ tiene un hijo/hija ya establecidos de manera permanente en EUA.

${ }^{7}$ Consultar por ejemplo la Tasa de prevalencia delictiva por cada cien mil habitantes en https://www.inegi. org.mx/temas/victimizacion/. En el índice no se incluye el homicidio.
} 
sus recursos económicos les resultan insuficientes para reiniciar sus vidas. Por ello, también $42.3 \%$ afirmó que la decisión de regresar a Guanajuato era temporal y de ellos $35.6 \%$ definitivamente tiene intención de regresar a EUA en la primera oportunidad; mientras que $36.4 \%$ no piensa regresar por ahora, o nunca.

Como se verá más adelante, el migrante tiene más confianza en las instituciones públicas estadounidenses que en las mexicanas; al tiempo que su familia es la base de su vinculación comunitaria. Al preguntarles si confían en su familia mientras estaban en EUA 83\% dijo que sí, mientras que $94 \%$ lo hace estando en México. Con los amigos sucede lo contrario, la confianza en los amigos disminuye de $74 \%$ mientras vivían en Estados Unidos a $70 \%$ al regresar a México; mientras que la confianza en los vecinos aumentó de $51 \%$ al $68 \%$ al moverse de Estados Unidos a México. Ello se explica porque algunos encuentran que su forma de vida ya no es aceptada en la comunidad $(6 \%)$, mientras que $18 \%$ percibe que su familia o amigos les tienen envidia o quieren que les presten dinero.

$\mathrm{Al}$ ser interrogados sobre los principales problemas que han tenido que enfrentar desde su regreso, las respuestas fueron, fundamentalmente, relacionados con exclusión del mercado o una inclusión inadecuada al mismo, así como ilegitimidad de los distintos niveles de gobierno:

Tabla 1. ¿Cuáles han sido los principales problemas que han tenido que enfrentar desde su regreso? (señale todos los que apliquen)

\begin{tabular}{|l|l|}
\hline Salario bajo e insuficiente & $62.4 \%$ \\
\hline Corrupción e ineficiencia de las autoridades & $50.5 \%$ \\
\hline Miedo e inseguridad & $48.6 \%$ \\
\hline Falta de servicios de salud & $29.4 \%$ \\
\hline Me tardé en encontrar un trabajo & $27.5 \%$ \\
\hline $\begin{array}{l}\text { Problemas para arreglar papeles/falta de papeles para } \\
\text { conseguir trabajo }\end{array}$ & $24.8 \%$ \\
\hline No trabajo en lo que aprendí a hacer en Estados Unidos & $16.5 \%$ \\
\hline Me tratan mal en el trabajo/No me dan prestaciones & $15.6 \%$ \\
\hline
\end{tabular}

Fuente: Elaboración propia sobre una pregunta abierta en la que anotamos todas las respuestas mencionadas. La pregunta se realizó solamente a las personas migrantes retornadas (114 respuestas).

Los jóvenes entrevistados, al confrontar su pertenencia en la comunidad responden:

yo, desde que me acuerdo, [en Estados Unidos] pues siempre mis costumbres de México, mi familia, siempre se han quedado con esas costumbres. Pero pues sí, también adoptamos las costumbres de Estados Unidos, que vienen siendo los Thanks Givings, el Día de Gracias, y así se puede decir que estoy 
entre en medio de mexicano y gringo o estadounidense, como le quiera decir. Sí, no podría decir [que soy] 100\% mexicano, ni 100\% estadounidense (Arturo, entrevista en Apaseo el Alto, 23 de febrero de 2016).

Al precisar dicen que cuando le piden ayuda a Dios lo hacen en español, pero cuando insultan, leen y hacen cuentas, lo hacen en inglés. Ellos creen que parte de su diferencia con sus pares es que hablan con acento, que no conocen las comunidades y se pierden con facilidad; conocen poco los centros de reunión, o que hablar inglés les hace aparecer presumidos. Sus padres encuentran más difícil educar a sus hijos en México (16\%) y señalan también que sus hijos tienen problemas porque no hablan, ni escriben en español (5\%).

El sentido de pertenencia también se midió por las redes sociales y familiares, elementos que se presentan en la Tabla 2. Durante el tiempo de estancia en EuA aumentó el número de personas casadas, con hijos o con amigos en el vecino país. La familia o razones familiares fue la razón fundamental por la que se definió el regreso a su comunidad en $79 \%$ de los casos, la discriminación, el miedo a la deportación y la deportación $28.6 \%$ y la falta de trabajo y el retiro por edad $19.5 \% .{ }^{8}$ No hay que olvidar que, como señalamos en el punto anterior, solamente $10 \%$ de los consultados se movieron entre fronteras con sus familias o las llevaron una vez que el jefe de familia estuvo instalado en Estados Unidos.

Tabla 2. El sentido de pertenencia de las personas migrantes

\begin{tabular}{|c|c|c|c|c|c|c|}
\hline \multirow{2}{*}{ Pregunta } & \multicolumn{2}{|c|}{ Antes de migrar } & \multicolumn{2}{|c|}{ En EUA } & \multicolumn{2}{c|}{ Al regresar } \\
\hline & Sí & No & Sí & No & Sí & No \\
\hline ¿Está usted casado? & $54.9 \%$ & $45.1 \%$ & $65.2 \%$ & $34.8 \%$ & $83.9 \%$ & $16.1 \%$ \\
\hline ¿Tiene hijos? & $49.6 \%$ & $50.4 \%$ & $68.2 \%$ & $31.8 \%$ & $88.2 \%$ & $11.8 \%$ \\
\hline $\begin{array}{c}\text { ¿Tiene familia o } \\
\text { amigos en EUA? }\end{array}$ & $63.6 \%$ & $36.4 \%$ & $80.7 \%$ & $19.3 \%$ & $79.8 \%$ & $20.2 \%$ \\
\hline
\end{tabular}

Fuente: Elaboración propia. La pregunta se realizó solamente a las personas migrantes retornadas (de 114 a 109 respuestas).

\section{Inclusión/exclusión: calidad de vida y trabajo}

Los migrantes de retorno toman entre seis y 11 meses en incluirse al mercado mexicano. La diferencia salarial hace que refuercen la percepción de que su calidad de vida personal y familiar disminuye, por las condiciones en que se insertan al mercado laboral en México. Es importante considerar este punto porque, a pesar de la crisis económica global en la que se analiza normalmente el retorno (Sandoval \& Zúñiga, 2016), solamente $17.1 \%$ señala haberse quedado sin trabajo en Estados Unidos y que esta sea la razón del regreso.

\footnotetext{
${ }^{8}$ La pregunta aceptaba varias opciones de respuesta, por eso no suma $100 \%$.
} 
Tabla 3. Condiciones de inclusión de los migrantes en mercados multiespaciales

\begin{tabular}{|c|c|c|c|c|c|c|}
\hline & \multicolumn{2}{|c|}{ Antes de migrar } & \multicolumn{2}{|c|}{ En EUA } & \multicolumn{2}{c|}{ Al regresar } \\
\hline & Sí & No & Sí & No & Sí & No \\
\hline ¿Tiene casa propia? & $46.2 \%$ & $53.8 \%$ & $56.7 \%$ & $43.3 \%$ & $80.4 \%$ & $19.6 \%$ \\
\hline ¿Tiene un coche? & $27.4 \%$ & $72.6 \%$ & $51.4 \%$ & $48.6 \%$ & $68 \%$ & $32 \%$ \\
\hline ¿Tiene una cuenta de banco? & $7.3 \%$ & $92.7 \%$ & $26.4 \%$ & $73.6 \%$ & $37.5 \%$ & $62.5 \%$ \\
\hline ¿Usa créditos bancarios? & $7.4 \%$ & $92.6 \%$ & $11.2 \%$ & $88.8 \%$ & $29.9 \%$ & $70.1 \%$ \\
\hline ¿Tiene agua corriente dentro & $81.1 \%$ & $18.9 \%$ & $89.2 \%$ & $10.8 \%$ & $93.3 \%$ & $6.7 \%$ \\
\hline \begin{tabular}{c} 
de su casa? \\
\hline ¿Tiene luz dentro de su casa?
\end{tabular} & $82.9 \%$ & $17.1 \%$ & $87.4 \%$ & $12.6 \%$ & $94.3 \%$ & $5.7 \%$ \\
\hline ¿Su calidad de vida es mejor? & $21.2 \%$ & $78.8 \%$ & $69 \%$ & $31 \%$ & $75.7 \%$ & $24.3 \%$ \\
\hline ¿La calidad de vida de su familia & $23.8 \%$ & $76.2 \%$ & $70.5 \%$ & $29.5 \%$ & $77.6 \%$ & $22.4 \%$ \\
\hline es mejor? & & & & & \\
\hline
\end{tabular}

Fuente: Elaboración propia. La pregunta se realizó solamente a las personas migrantes retornadas (de 114 a 109 respuestas).

La baja en la calidad de vida tiene relación con que las personas retornadas también se incluyen en trabajos informales en México. Este último punto se asume porque a los únicos programas sociales gubernamentales a los que buscan acceder son los que fueron hasta 2019 el Programa Oportunidades ${ }^{9}(23.2 \%)$ y el Seguro $\operatorname{Popular}^{10}(95.3 \%)$, lo cual es un reflejo de que la calidad y seguridad del empleo no es óptima. Los migrantes retornados que cuentan con un empleo formal lo definimos por la afiliación al Instituto Mexicano del Seguro Social (IMSs) (13.6\%), al Instituto de Seguridad y Servicios Sociales de los Trabajadores del Estado (ISSSTE) (2.4\%) o al Instituto de Seguridad Social del Estado de Guanajuato (ISSEG) (0.2\%); mientras que $10.5 \%$ no cuenta con ningún tipo de seguridad social, a pesar de contar con un empleo. Aun cuando $41 \%$ recibió algún tipo de capacitación en Estados Unidos, $82 \%$ asegura que esta le ha servido poco o nada para reinsertarse al mercado laboral nacional. A pesar de ello, como se muestra en la Tabla 3, la migración les permitió contar con una casa propia, un automóvil, utilizar cuentas y créditos bancarios. Como veremos más adelante, la sensación de contar con una mejor calidad de vida tiene más que ver con el reconocimiento comunitario, el sentido de pertenencia vis-à-vis, la discriminación vivida, y el regresar con su familia, aunque sus ingresos hayan disminuido.

Las personas que recibieron ayuda de un familiar o amigo para encontrar un trabajo conforman $44.5 \%$ y ninguna se acercó al gobierno para buscar información para encontrar trabajo, acceder a recursos económicos para iniciar un proyecto

\footnotetext{
${ }^{9}$ Oportunidades era un programa de desarrollo humano que garantizaba fondos a familias que vivían en pobreza y pobreza externa (\$1 USD por día) para asegurar acceso a condiciones mínimas de sobrevivencia. El recurso económico se les otorgaba mensualmente a las mujeres -en tanto jefas de familia- y estaba condicionado a que los hijos asistieran a la escuela -el apoyo se incrementaba conforme los hijos avanzaban en el sistema educativo - así como a revisiones semestrales de salud.

${ }^{10}$ Seguro Popular era un programa creado en 2002 para ofrecer acceso a atención médica a personas trabajando en los mercados informales y, por esa razón, excluidos de los programas de seguridad social. El pago de servicios está en relación directa con el ingreso de las familias. El programa ayuda a mitigar los gastos médicos catastróficos a las personas que viven por debajo de los niveles de pobreza.
} 
productivo o para actualizar sus papeles de identidad. Todas esas actividades las realizan a través de sus ahorros propios, de relacionarse con personas migrantes que conocen cómo se trabaja en el mercado estadounidense, que establecieron un negocio en su comunidad, o tras preguntar a sus amigos en dónde se renuevan las tarjetas de identidad del Instituto Nacional Electoral (INE), se obtiene una Clave Única de Registro de Población (CURP) o las actas de nacimiento. ${ }^{11}$

\section{Participación/apatía: la casi nula participación en organizaciones sociales}

La apatía es la característica fundamental de las personas migrantes que, en general, están mal organizadas y participan poco en organizaciones sociales tanto en México, como en Estados Unidos (Tabla 4).

Tabla 4. Participación multiespacial de las personas migrantes

\begin{tabular}{|c|c|c|c|c|c|c|}
\hline \multirow[t]{2}{*}{ Pregunta } & \multicolumn{2}{|c|}{ Antes de migrar } & \multicolumn{2}{|c|}{ En EUA } & \multicolumn{2}{|c|}{$\mathrm{Al}$ regresar } \\
\hline & Sí & No & Sí & No & Sí & No \\
\hline ¿Participa en un sindicato? & $7.6 \%$ & $92.4 \%$ & $7.5 \%$ & $92.5 \%$ & $9.4 \%$ & $90.6 \%$ \\
\hline $\begin{array}{c}\text { ¿Participa en organizaciones de } \\
\text { su iglesia? }\end{array}$ & $5.6 \%$ & $94.6 \%$ & $5.5 \%$ & $94.5 \%$ & $11 \%$ & $89 \%$ \\
\hline $\begin{array}{l}\text { ¿Participa en la organización } \\
\text { de las fiestas patronales? }\end{array}$ & $23.2 \%$ & $76.8 \%$ & $19.8 \%$ & $80.2 \%$ & $43.7 \%$ & $56.3 \%$ \\
\hline $\begin{array}{c}\text { ¿Participa en alguna asociación } \\
\text { deportiva? }\end{array}$ & $15 \%$ & $85 \%$ & $15.6 \%$ & $84.4 \%$ & $15.6 \%$ & $84.4 \%$ \\
\hline ¿Participa en un club migrante? & $0 \%$ & $100 \%$ & $4.7 \%$ & $95.3 \%$ & $3.8 \%$ & $96.2 \%$ \\
\hline ¿Vota en las elecciones? & $43 \%$ & $57 \%$ & $3 \%$ & $97 \%$ & $58.9 \%$ & $41.1 \%$ \\
\hline
\end{tabular}

Fuente: Elaboración propia. La pregunta se realizó solamente a las personas migrantes retornadas (de 114 a 109 respuestas).

La única participación comunitaria que los migrantes mantienen a distancia es la organización de las fiestas patronales. Desde Estados Unidos participaban enviando dinero (20\%), mientras que en México $44 \%$ lo hace en efectivo y en especie. Sin embargo, al regresar hay un leve incremento $(11 \%)$ en la participación en alguna asociación religiosa, cuando solamente cerca $6 \%$ lo hacía en EUA. La intervención en asociaciones deportivas, básicamente en equipos de futbol soccer, tanto en México, como en Estados Unidos, se mantiene en 16\%. La participación en un club migrante se da en 4\%, y solamente durante su estancia en el país del norte. La participación en las elecciones aumenta algunos puntos al reestablecerse en México.

\footnotetext{
11 Según los datos proporcionados por el Instituto de Atención al Migrante Guanajuatense y sus Familias, entre 2014 a 2017 se otorgó alguna forma de apoyo económico a 177 migrantes deportados que lo solicitaron y se encontraron varados en alguno de los puntos de internación de la frontera norte de México. A través del programa de Inversión Migrante se crearon solamente 120 microproyectos productivos para migrantes retornados.
} 


\section{Reconocimiento/rechazo: del voto a la confianza de los vecinos}

La sensación de ser aceptado por su comunidad se incrementa de manera importante al regresar a México (Tabla 5). Aunque la mayor parte de las respuestas sobre la experiencia de vida en Estados Unidos refleja el sentimiento de rechazo y discriminación experimentados por las personas durante su estadía en ese país. La percepción de ser reconocidos o rechazados en sus comunidades se midió (véase Tabla 5) con preguntas como ¿usted cree que la gente votaría por usted? A lo que 33\% de los que regresaron dijeron que sí, contra 14\% que lo aseguró mientras vivía en Estados Unidos. Del mismo modo, preguntamos si sus vecinos les dejarían cuidar a sus hijos en caso de necesidad, respuesta que pasó de 51\%, mientras vivió en Estados Unidos a 73\%, ahora que vive en México. Ellos tampoco muestran confianza en sus vecinos estadounidenses, ya que $42 \%$ hubiese solicitado a sus vecinos cuidar a sus niños; en México $63 \%$ dejaría a sus niños con sus vecinos. Estas respuestas muestran que se sienten reconocidos y como personas de confianza en México, aunque ellos sean más cautelosos en reconocer a sus vecinos o amigos como personas dignas de confianza en su propio país.

Tabla 5. Reconocimiento de las personas migrantes en las comunidades vividas

\begin{tabular}{|c|c|c|c|c|c|c|}
\hline \multicolumn{1}{c|}{ Pregunta } & \multicolumn{2}{|c|}{ Antes de migrar } & \multicolumn{2}{|c|}{ En EUA } & \multicolumn{2}{c|}{ Al regresar } \\
\hline & Sí & No & Sí & No & Sí & No \\
\hline $\begin{array}{c}\text { ¿Cree que la gente de su } \\
\text { comunidad lo aprecia? }\end{array}$ & $71.8 \%$ & $28.2 \%$ & $54.8 \%$ & $45.2 \%$ & $78.1 \%$ & $21.9 \%$ \\
\hline $\begin{array}{c}\text { ¿Sus vecinos le dejarían } \\
\text { cuidar a sus hijos en caso de } \\
\text { necesidad? }\end{array}$ & $60.4 \%$ & $39.6 \%$ & $50.5 \%$ & $49.5 \%$ & $72.7 \%$ & $27.3 \%$ \\
\hline $\begin{array}{c}\text { ¿Usted dejaría que sus } \\
\text { vecinos cuiden a sus hijos en } \\
\text { caso de necesidad? }\end{array}$ & $57.1 \%$ & $42.9 \%$ & $41.6 \%$ & $58.4 \%$ & $63.4 \%$ & $36.6 \%$ \\
\hline $\begin{array}{c}\text { ¿Usted cree que la gente } \\
\text { votaría por usted? }\end{array}$ & $17.2 \%$ & $82.8 \%$ & $13.8 \%$ & $86.2 \%$ & $33.3 \%$ & $66.7 \%$ \\
\hline $\begin{array}{c}\text { ¿Usted cree que sus vecinos } \\
\text { lo ven como a un igual? }\end{array}$ & $57.7 \%$ & $42.3 \%$ & $46 \%$ & $54 \%$ & $69 \%$ & $31 \%$ \\
\hline $\begin{array}{c}\text { ¿Usted se siente igual a sus } \\
\text { vecinos? }\end{array}$ & $67 \%$ & $33 \%$ & $58.8 \%$ & $41.2 \%$ & $70.6 \%$ & $29.4 \%$ \\
\hline
\end{tabular}

Fuente: Elaboración propia. La pregunta se realizó solamente a las personas migrantes retornadas (109 respuestas).

\section{Legitimidad/ilegitimidad: invisibles para el gobierno en México/tolerados en Estados Unidos}

Como muestra la Tabla 1 más arriba, el primer problema a enfrentar es la caída en el nivel de vida: los salarios en México difícilmente se acercan a los percibidos en el norte, aunque mantienen trabajos sin prestaciones y en situación inestable. El segundo problema es la corrupción e ineficiencia de las autoridades para facilitarles acceso a los 
programas sociales, sea porque les piden dinero para "facilitar los trámites", sea porque el tiempo que hay que invertir en acceder a ellos los desmotiva. El tercer problema enfrentado por las personas migrantes retornadas es el miedo y la inseguridad. En general, responde a las condiciones que tienen que enfrentar en su vida cotidiana en donde son víctimas de robos, asaltos y pérdida de los recursos ganados en sus trabajos.

Tabla 6. Legitimidad de las instituciones para las personas migrantes

\begin{tabular}{|c|c|c|c|c|c|c|}
\hline Pregunta & Antes de migrar & \multicolumn{2}{|c|}{ En EUA } & \multicolumn{2}{c|}{ Al regresar } \\
\hline & Sí & No & Sí & No & Sí & No \\
\hline ¿Confía usted en el gobierno? & $25.5 \%$ & $74.5 \%$ & $29.3 \%$ & $70.7 \%$ & $18.5 \%$ & $81.5 \%$ \\
\hline ¿Confía usted en su familia? & $88.5 \%$ & $11.5 \%$ & $83.3 \%$ & $16.7 \%$ & $93.5 \%$ & $6.5 \%$ \\
\hline ¿Confía usted en sus amigos? & $78.1 \%$ & $21.9 \%$ & $73.6 \%$ & $26.4 \%$ & $70.4 \%$ & $29.6 \%$ \\
\hline ¿Confía usted en sus vecinos? & $67.6 \%$ & $32.4 \%$ & $50.5 \%$ & $49.5 \%$ & $67.6 \%$ & $32.4 \%$ \\
\hline ¿Su seguridad personal está & $56.9 \%$ & $43.1 \%$ & $57.6 \%$ & $42.4 \%$ & $67 \%$ & $33 \%$ \\
\hline \begin{tabular}{c} 
mejor? \\
\hline
\end{tabular} & & & & & \\
\hline
\end{tabular}

Fuente: Elaboración propia. La pregunta se realizó solamente a las personas migrantes retornadas (de 114 a 109 respuestas).

Para reafirmar esta última pregunta hicimos un comparativo entre la percepción de su seguridad personal antes de migrar, mientras vivió en Estados Unidos y al regresar. El registro (Tabla 6) muestra un incremento en la confianza en el gobierno de Estados Unidos, que disminuye al regresar a México, esto a pesar de que viven en condiciones irregulares. En este punto hay que considerar la diferencia de la respuesta espontánea dada a esta pregunta: "Allá las cosas si funcionan”, "allá las personas hacen su trabajo sin pedirte dinero". La baja legitimidad del gobierno queda en desventaja en México, en Guanajuato y en los municipios; la ilegitimidad del gobierno federal, estatal y municipal se manifiesta antes de partir y al regresar. ${ }^{12}$

$\mathrm{Al}$ regresar, las personas migrantes perciben un cambio en la forma como son recibidos en instituciones de salud. Esto a pesar de que $79.5 \%$ de los migrantes retornados no contaban con algún tipo de seguridad social mientras trabajaron en Estados Unidos y solamente $55.2 \%$ de los encuestados consideraban accesibles los servicios de salud mientras vivieron en ese país. Las respuestas sobre las principales dificultades encontradas en los servicios de salud estadounidense fueron: ellos no tenían dinero para acudir (36\%), no tener papeles $(66.7 \%)$, le negaban el servicio (38.9\%) y el patrón no lo pagaba (22.2\%). A pesar de ello, al pedirles que comparen el servicio y acceso a los sistemas de salud en Estados Unidos y en México 63\% manifestó preferir los servicios de salud en el norte. Las respuestas espontáneas con las que explican esta preferencia fueron: "ofrecen mejor servicio", "tienen mejores instalaciones", "cuentan con personal capacitado", "allá sí te atienden, aunque no tengas papeles", "te atienden, aunque no traigas dinero y luego te cobran de acuerdo con tus ingresos", "allá les importa tu vida" y "allá te tratan con respeto".

\footnotetext{
${ }^{12}$ Este tema también es trabajado por Tse $(2019 ; 2020)$ y Mateos (2020), sobre la invisibilidad que tienen los migrantes en la política pública dirigida a los que regresaron masivamente en la última década.
} 
Las principales quejas sobre las instituciones de salud mexicanas fueron: "tardan mucho en atender", "están lejos de mi localidad", "nunca tienen medicinas", "el servicio es malo y no te atienden si no traes dinero". Lo fundamental de la relación entre las personas y las instituciones de salud es que en Estados Unidos su vida sí es importante y son tratados con respeto, "aunque sean ilegales", y en México, siendo ciudadanos, les niegan el servicio por ser pobres. Al comparar las respuestas, no podemos dejar de resaltar que, aunque sean indocumentados, pueden recibir el servicio porque allá "les importa tu vida", "te tratan con respeto" y "dan servicio, aunque no tengan papeles o dinero". Esto refuerza la legitimidad del Estado, en este caso Estados Unidos, frente a una comunidad que se asienta en su territorio de manera irregular, a pesar de haber sido víctima de discriminación, redadas y políticas antiinmigrantes.

\section{Discusión}

Al discutir los resultados de este ejercicio, hay que señalar que el trabajo de campo se realizó en 10 comunidades de tres municipios en el sur de Guanajuato. Esto afecta la definición y contextualización de comunidad nacional, subnacional y local, ya que mientras la primera es la que está concebida en la noción de cohesión social presentada arriba, las tendencias que se muestran en este texto se encontraron a nivel local. ${ }^{13}$ La encuesta se diseñó con la idea original de utilizar la que consideramos la definición más acabada de cohesión social para tratar de medir los impactos de la migración de retorno en las comunidades de Guanajuato. Al analizar la migración desde una perspectiva histórica, detectamos las estructuras de vinculaciones comunitarias simultáneas y permanentes en dos espacios geográficos como parte del proceso individual vivido por los migrantes. Esto permite establecer la hipótesis de que los elementos y valores que se comparten dentro de una sociedad bien ordenada pueden existir y mantenerse transnacionalmente en comunidades donde conviven poblaciones móviles y estáticas, como son las poblaciones estudiadas en Guanajuato. El tema cobra importancia para destacar las tendencias e hipótesis que podrían empezar a detonar una agenda de investigación sobre la existencia o no de estructuras de vinculaciones que mantienen comunidades cohesionadas trasnacionalmente, con base en los hallazgos encontrados. ${ }^{14}$

Hecha esta advertencia, la primera tendencia detectada es que los grupos comunitarios móviles han creado una dinámica de valores compartidos en las que mantienen vínculos cotidianos entre fronteras. Es decir, los resultados parecen indicar que el compromiso social y los valores compartidos de estos grupos móviles se viven y manifiestan de manera transnacional. Es decir, que las dimensiones que componen

\footnotetext{
${ }^{13}$ Es importante agradecer el cuidadoso trabajo de revisión realizado por los dictaminadores que nos obligan a reflexionar sobre estos problemas epistemológicos y, sobre todo, para destacar los límites de nuestros hallazgos y tomarlos en cuenta en trabajos futuros.

${ }^{14}$ Ciertamente, para continuar la agenda desde una perspectiva transnacional se deben de incluir las preguntas sobre el papel de las redes sociales y la comunicación simultánea y elementos que ayuden a profundizar más sobre la temática transnacional, también hacer entrevistas a profundidad con personas retornadas que expliquen sus razones de participación comunitaria vis-à-vis en organizaciones sociales, etcétera. El tema de las relaciones transnacionales surgió al analizar los resultados, no al diseñar la encuesta que buscaba explorar el estado de la cohesión social de las comunidades con grupos migrantes retornando a ellas. La autora agradece los comentarios de los revisores anónimos.
} 
la cohesión social se mantienen simultáneamente en el tiempo, aunque en espacios distintos. En términos de inclusión, los migrantes se incluyen de manera informal en el mercado laboral estadounidense, pero sus ingresos se gastan en forma de remesas por sus familias en otro; en el que participan en la organización de sus fiestas patronales en un país, cuando la fiesta se celebra en otro; son reconocidos como miembros de la comunidad en los dos lados de la frontera - aceptados o discriminados- pero mantienen una presencia activa en las mismas.

Esto no quiere decir que no vivan condiciones de vulnerabilidad. Ya que mientras se insertan en los nichos de mercado menos calificados de Estados Unidos, sus familias en México tienen una calidad material de vida mejor. La migración les permite adquirir casa, automóvil, tierras y animales, entre otros bienes. Al regresar, tienen la percepción de que la calidad de vida material de ellos y sus familias disminuye debido a la baja calidad de los trabajos en los que se insertan, a pesar de que, en su mayoría, tienen casa propia, coche, educación financiera y pudieron apoyar la educación y mejorar la salud de sus hijos. Estos aspectos implicarían una calidad de vida superior a las de sus pares comunitarios, mientras sacrificaron su calidad de vida social. El reconocimiento y participación comunitaria, intragrupal, o familiares se mantienen por contactos telefónicos frecuentes, envío de remesas, financiamiento de las fiestas patronales, etcétera. La participación en clubes de futbol soccer, la confianza en sus vecinos, el reconocimiento y participación comunitario es menos frecuente, pero no nulo. En términos de pertenencia a la comunidad política y en la legitimidad de las instituciones, el porcentaje cae, pero con un mayor reconocimiento a la efectividad institucional del gobierno de Estados Unidos.

El sentido de pertenencia, reconocimiento y seguridad se incrementa al vivir en México, lo que fortalece el tejido social de las comunidades de retorno. Se puede afirmar que las redes sociales y familiares con las que cuentan los migrantes en Estados Unidos son las que les ayudan a sostener la migración; la densidad de esas mismas redes en Guanajuato son las que ayudan a soportar la reintegración en las comunidades de origen y retorno, las que apoyan la reinserción de ellos y de sus hijos en sus comunidades. Los lazos sociales y afectivos son los que parecen determinar la calidad de vida social, preparar y justificar el retorno al origen, hasta que las circunstancias económicas apremien y presionen por una nueva salida.

Como han constatado otros trabajos citados, los migrantes han estado presentes en las políticas públicas cuando están en Estados Unidos, y ausentes cuando regresan a México (Tse, 2020; Mateos, 2020). La legitimidad de un gobierno receptor que los ve como un riesgo y uno emisor que los ignora se percibe en la muy pobre visión que tienen las personas migrantes en las instituciones públicas y en el gobierno. En el caso de los que han regresado, se suma el maltrato que reciben al transportar sus cosas, asistir a los servicios de salud públicos o a la falta de acceso a algunos servicios, la dificultad para lograr documentos de identificación para obtener trabajos, entre otros. Todos estos elementos reducen la legitimidad de las autoridades mexicanas frente a los migrantes retornados, que en algunos casos expresan mayor confianza en las instituciones estadounidenses a las que perciben más empáticas al interesarse por ellos o su vida, a pesar de haber vivido un ambiente de acoso y sin papeles en Estados Unidos, como se refleja en las respuestas espontáneas citadas más arriba.

La calidad de vida social de los migrantes experimenta un incremento en la sensación de seguridad, reconocimiento y participación comunitaria al volver a casa. Contrasta también con el cambio que representa la percepción de seguridad mientras 
vivían en condiciones irregulares en comunidades estadounidenses que discriminan en contra de ellos a pesar de contratar su trabajo, que se refleja en la percepción del ambiente antiinmigrante y de las redadas de las que algunos fueron víctimas. Ni el gobierno de México, ni el gobierno de Guanajuato les ofrecen las garantías mínimas que el Estado les debe de otorgar para garantizar una vida sin conflictos, que se incluye en la definición de legitimidad analizada como parte integrante de la cohesión social. Finalmente, tanto en México como en Estados Unidos se trata de grupos sociales vulnerables que se reintegran a comunidades institucionalmente débiles, en la medida en que cuentan con una participación baja en organizaciones sociales.

\section{Conclusiones}

Los elementos aquí presentados nos permiten concluir dos puntos: primero, dentro de las comunidades guanajuatenses se han conformado grupos sociales que han organizado su vida social y económica compartiendo valores y compromisos comunitarios que alternan espacialmente. Esto no altera la calidad de su vida comunitaria, ni el reconocimiento mutuo, ni la participación en actividades sociales. Por el contrario, encontramos que la simultaneidad temporal y espacial se refuerza al vincular algunas actividades económicas, sociales y culturales en Estados Unidos y otras en México. Lo que en el fondo refuerza vínculos y sentidos de pertenencia multiespacial basados en redes sociales que, paradójicamente, pareciera situarlos en una desventaja cívica (Vila Freyer, 2019; Vila Freyer, 2017). Son las instituciones políticas débiles las que afectan de manera negativa las relaciones entre grupos migrantes y las distintas maneras de gestionar las relaciones sociales entre los ciudadanos. Lo que Portes y Vickstrom (2012, p. 100) definen como comunidades "donde la solidaridad mecánica de las familias extendidas y las redes tribales mantiene una apariencia de orden frente a instituciones coordinadoras débiles o ausentes".

Segundo, hasta ahora, las discusiones sobre cómo la migración impacta en las sociedades de acogida asume efectos negativos de la migración en la cohesión social de las comunidades receptoras. Los migrantes son vistos como un riesgo al no compartir los elementos culturales, valores o sentido de solidaridad con la sociedad de acogida. Hay autores que hacen énfasis en los aspectos económicos y no socioculturales los que explicarían las dificultades de participación en las sociedades de acogida (Ratcliffe, 2012). Portesy Vickstrom (2012) aseguran que el conflicto que pudiese crearse por los migrantes en las sociedades de acogida se logra reducir por las instituciones políticas fuertes que lo gestionan o que, nosotros agregaríamos, lo reaniman. Sin embargo, al analizar estos elementos desde el punto de vista de las sociedades emisoras encontramos relaciones comunitarias mucho más densas y multiespaciales, y en buena medida autorreguladas, que refuerzan el sentido comunitario y la solidaridad mecánica en las mismas. Es como si la migración dificultase los procesos de complejidad social en las comunidades migrantes, reforzando y manteniendo las relaciones tradicionales en las mismas. No se trata solo de la inclusión en el mercado, sino en la densidad de las redes sociales con las que están vinculados lo que fragmenta el tejido social, elemento que, a su vez, sirve para explicar y justificar la emigración, en primer lugar.

De ahí deriva la necesidad de desarrollar una agenda de investigación que analice y reflexione sobre el estado de la cohesión social en Guanajuato, que requiere 
necesariamente comparar las condiciones de las poblaciones móviles e inmóviles, para medir el verdadero impacto de la migración en su tejido social.

\section{Agradecimientos}

El trabajo de campo para este proyecto fue financiado por la Secretaría de Educación Pública que, a través del Programa para el Desarrollo del Personal Docente (ProdepApoyo a nuevos PTCs). En el levantamiento de las encuestas participaron los estudiantes Airton Ramírez, Mónica Ruíz, Fernanda Sauz y Plácido Sierra. En la recopilación y análisis de los datos tuve el apoyo de Ma. Teresa Lara, Isamar Ramírez y Pablo León Hacha. La autora agradece a todos.

\section{Referencias}

Anderson, B. (2019). New directions in migration studies: towards methodological de-nationalism. Comparative Migration Studies, 7(36). https://doi.org/10.1186/ s40878-019-0140-8

Consejo Nacional de Población (Conapo). (2001). Índice de Intensidad Migratoria México Estados Unidos. Conapo, Secretaría de Gobernación.

Consejo Nacional de Población (Conapo). (2012). Índice de Intensidad Migratoria México Estados Unidos. Conapo, Secretaría de Gobernación.

Coleman, J. (1988). Social capital in the creation of human capital. The American Journal of Sociology, 94 (Supplement: Organizations and institutions: sociological and economic approaches to the analysis of social structure), S95-S120.

Dickes, P., Valentova, M. \& Borsenberger, M. (2011). A multidimensional assessment of social cohesion in 47 European countries. LISER Working Papers (2011-07). https://liser.elsevierpure.com/en/publications/a-multidimensional-assessment-of-social-cohesion-in-47-european-c

Durand, J. (2016). Historia mínima de la migración México-Estados Unidos. El Colegio de México.

Durand, J. \& Massey, D. (2003). Clandestinos. Migración México-Estados Unidos en los albores del siglo XXI. Universidad Autónoma de Zacatecas/Miguel Ángel Porrúa.

Eckstein, S. (2015). Deepening and broadening transnational immigration analyses: commentary on Roger Waldinger's The Cross-Border Connection. Ethnic and Racial Studies, 38(13), 2291-2298. http://dx.doi.org/10.1080/01419870.2015.1 058501

Glick Schiller, N., Basch, L. \& Blanc-Szanton, C. (1992). Transnationalism: A new analytic framework for understanding migration. Annals of the New York Academy of Sciences, 645(1), 1-24. https://doi.org/10.1111/j.1749-6632.1992.tb33484.x

Guevara Sanginés, M. (2017). Guanajuato: territorio de inmigrantes. Siglos Xvi-XviII. Ciencias Sociales y Humanidades. Revista Centroamericana de Investigación y Postgrado, 4(1), 45-58. https://digi.usac.edu.gt/ojsrevistas/index.php/csh/article/ view/461 
Jenson, J. (1998). Mapping social cohesion: the state of Canadian research (CPRN study núm. F/03). Renouf Publishing. http://www.cccg.umontreal.ca/pdf/CPRN/CPRN_ F03.pdf

Jenson, J. (2010). Defining and measuring social cohesion. Commonwealth Secretariat and United Nations Research Institute for Social Development.

Jenson, J. \& Saint-Martin, D. (2003). New routes to social cohesion? Citizenship and the social investment state. The Canadian Journal of Sociology/Cahiers Canadiens de Sociologie, 28(1), 77-99.

López Sánchez, E. (2016). Crisis en la enseñanza de la metodología cualitativa. Andamios 13(31), 109-127. http://dx.doi.org/10.29092/uacm.v13i31.429

Mateos, P. (2020). Mexican-u.s asymmetrical diaspora policies in the age of return migration. Migration Letters, 17(1), 147-153. https://doi.org/10.33182/ ml.v17i1.866

Moctezuma, M. (2013). Retorno de Migrantes a México. Su reformulación conceptual. Papeles de Población, 19(77), 149-175.

Portes, A. \& Vickstrom, E. (2012). Diversidad, capital social y cohesión. RES, (17), 83107. http://www.fes-sociologia.com/files/res/17/05.pdf

Ratcliffe, P. (2012). 'Community cohesion': reflections on a flawed paradigm. Critical Social Policy, 32(2), 262-281. https://doi.org/10.1177\%2F0261018311430455

Sandoval, R. \& Zúñiga, V. (2016). ¿Quiénes están retornando de Estados Unidos a México?: una revisión crítica de la literatura reciente (2008-2015). Mexican Studies/ Estudios Mexicanos, 32(2), 328-356. https://doi.org/10.1525/mex.2016.32.2.328

Tse, V. (2019). Dreams of transnational social protection: The case of Mexican policies towards youth migration. The Migration Conference, 2019. Book of abstracts and programme. Transnational Press London.

Tse, V. (2020). Dreams of transnational social protection: Youth returnees in Mexico. En A. Vila-Freyer \& M. G. Özerim (Eds.), Young migrants: vulnerabilities, boundaries, protection and integration (pp. 35-55). Transnational London Press.

Vila Freyer, A. (2015). Who are the returnees? Return migration in Guanajuato 20052010: Three scenarios in a rural context. International Journal of Humanities and Social Sciences, 5(11), 50-59. http://www.ijhssnet.com/journals/Vol_5_No_11_ November_2015/6.pdf

Vila Freyer, A. (2017). Pertenencias múltiples e identidades compuestas en un contexto norteamericano. Exploraciones a partir de la trayectoria migratoria de cuatro jóvenes en el sur de Guanajuato. Norteamérica, 12(1), 53-78. http://dx.doi. org/10.20999/nam.2017.a002

Vila Freyer, A. (2019). Pertenencias múltiples e identidades compuestas en viejas y nuevas migraciones en México. Cahiers des Ameriques Latines, 91(2), 133-152. https://doi.org/10.4000/cal.9702 


\begin{abstract}
Ana Vila Freyer
Mexicana. Doctora en ciencia política por la Université de Montréal (Canadá), maestra en sociología política por el Instituto Mora (Conacyt/México). Profesora-investigadora de la Universidad Latina de México. Integrante del Sistema Nacional de Investigadores. Miembro del Comité Científico y responsable del Capítulo de Juventud Migrante de The Migration Conference. Líneas de investigación: jóvenes migrantes, migración de retorno en Guanajuato, migración de tránsito por Guanajuato. Publicaciones recientes: Vila Freyer, A. (2020). Atado al sueño de ser adoptado por un país que no es el tuyo: La definición de Dreamer desde la perspectiva de los jóvenes migrantes, Norteamérica, 15(2), http://dx.doi.org/10.22201/cisan.24487228e.2020.2.435; Vila Freyer, A. \& Özerim, G. M. (2020). Young migrants, vulnerabilities, boundaries, protection, and integration. Transnational Press London.
\end{abstract}

